\title{
Lidocaine infusion for continuous interscalene nerve block: Is there evidence for local and systemic benefits?
}

\author{
Vivian H. Y. Ip, MBChB • Ban C. H. Tsui, MD
}

Received: 28 October 2014/Accepted: 11 November 2014/Published online: 20 November 2014

(C) Canadian Anesthesiologists' Society 2014

\section{To the Editor,}

We read with interest the case report by BurckettSt.Laurent et al. ${ }^{1}$ describing ultrasound-guided superior trunk block as an alternative approach to interscalene block with the potential to reduce inadvertent phrenic nerve blockade. We applaud their excellent work and herein describe an approach we have used to minimize adverse respiratory effects following interscalene block.

A 54-yr-old male (body mass index 52.2) was scheduled for a left shoulder hemiarthroplasty. He had a history of well-controlled asthma on salbutamol and also suffered obstructive sleep apnea, which was managed at night with continuous positive airway pressure. He was taking Oxycocet every six hours along with diclofenac to manage chronic arthritic back and knee pain. The patient consented to undergo the interscalene block after being apprised of the block's susceptibility to phrenic nerve blockade consequent with its analgesic benefits.

A left interscalene MultiSet 211156-40E catheter-overneedle assembly (Pajunk GmbH; Geisingen, Germany) was inserted using the conventional technique with an out-ofplane approach between the C5 and C6 roots. Nevertheless, the catheter was not injected until five minutes before induction of general anesthesia, at which time $5 \mathrm{~mL}$ of local anesthetic (0.5\% ropivacaine: $0.125 \%$ bupivacaine 1:1) were administered. The intraoperative period was uneventful, and the patient was comfortable in the postanesthesia care unit with a good sensory block of the C5-8 regions. To provide the volume necessary for adequate blockade, an infusion of $0.4 \%$ lidocaine at 3

V. H. Y. Ip, MBChB (凶) • B. C. H. Tsui, MD

Department of Anesthesiology and Pain Medicine, University of Alberta Hospital, Edmonton, AB, Canada

e-mail: hip@ualberta.ca
$\mathrm{mL} \cdot \mathrm{hr}^{-1}$ was initiated with pre-planned hourly $3-\mathrm{mL}$ bolus doses throughout the time the catheter remained in place. We reasoned that, in the event of respiratory compromise, the lidocaine infusion could be stopped with a relatively quick offset compared with other local anesthetics.

Postoperatively, the patient was comfortable with a visual analogue scale pain score $<3$. No symptoms or signs of respiratory compromise or local anesthetic toxicity were observed. The only other pain control used was his usual home analgesics. We discontinued the infusion on postoperative day two. Interestingly, the patient started experiencing more back and knee pain less than two hours after discontinuing the infusion.

Several interesting aspects emerged from this case. Firstly, injecting local anesthetic through the interscalene catheter just prior to induction may avoid subjective effects from phrenic nerve blockade. Secondly, if respiratory compromise should occur, the use of lidocaine may avoid a prolonged course. Thirdly, we did not need to increase the local anesthetic infusion, suggesting no clinically significant catheter migration for at least 48 hours. Lastly, the lidocaine infusion appeared to have relieved the patient's chronic arthritic pain. Indeed, an intravenous lidocaine infusion has been used to control chronic pain owing to its local anesthetic and anti-inflammatory properties. $^{2,3}$ Future studies will evaluate the use of lidocaine for nerve block infusion and will assess its systemic analgesic effects.

Funding Ban Tsui is supported by a Clinical Scholar Award from the Alberta Heritage Foundation for Medical Research (AHFMR). Dr. Tsui's research is supported by the Canadian Anesthesia Research Foundation. The Pajunk MultiSet 211156-40E is modified and redesigned by Ban Tsui. Dr. Tsui also has a patent-licensing agreement with Pajunk GmbH. 


\section{References}

1. Burckett-St.Laurent D, Chan V, Chin KJ. Refining the ultrasound-guided interscalene brachial plexus block: the superior trunk approach. Can J Anesth 2014; DOI:10.1007/s12630014-0237-3.
2. Caracas HC, Maciel JC, Martins PM, de Souza MM, Maia LC. The use of lidocaine as an anti-inflammatory substance: a systematic review. J Dent 2009; 37: 93-7.

3. Vigneault $L$, Turgeon $A F$, Cote $D$, et al. Perioperative intravenous lidocaine infusion for postoperative pain control: a meta-analysis of randomized controlled trials. Can J Anesth 2011; 58: 22-37. 